\title{
e-17. Diagnostic Value of Vertebral Angiography in Cases of the Posterior Fossa Tumors
}

\author{
Masahiro MizukamI, Toru Mine and Tatsuyuki Kudo \\ Division of Neurological Surgery, Department of Surgery, \\ School of Medicine, Keio University
}

Vertebral angiography has been considered to be of little diagnostic value in the localization of posterior fossa tumors. Pneumoventriculography is therefore generally accepted to be of great importance.

The variations in the course of the vessels in the posterior fossa and their correlative relationship to the adjacent structures were studied at autopsy by dissection and at vertebral angiography in living subjects.

Despite numerous variations, it is possible to select configurations of the posterior inferior cerebellar artery, the precentral cerebellar vein and the medial inferior cerebellar vein which can be considered as standard on vertebral angiography.

These vessels moreover are easily identified on angiograms and the caurses of them are intimately related to the adjacent structures such as the medulla, the tonsils, the aqueduct, the posterior aspects of fourth ventricle, the vermis and the medial inferior aspect of cerebellar hemisphere.

Displacements and deformities of these vessels may therefore be interpreted in terms of the typical configurations and are of considerable importance in the recognition and localization of expanding lesions.

In conclusion percutaneous retrograde brachial angiography is easier and less dangerous than pneumoventriculography in establishing the diagnosis and should be more highly appreciated and recommended.

\section{e-18. Diagnosis and Treatment of Incisural Tumors}

\author{
Kenzo Matsuoka, Tohru Uozumi, Mitsumasa Kano, Tsukasa Kishida, \\ Kazuyasu NaKao, Kazuhiko NonaKa, Yuji HamanaKa and \\ Tsutomu Yasumitsu \\ 1st Department of Surgery, Osaka University, Medical School
}

There are some space-occupying lesions which have their origin at or adjacent to the incisura tentorii. A few authors have attempted to specify these lesions under a heading of "incisural space-occupying lesions", because, both in the diagnosis and in the neurosurgical treatment, special attention should be paid to these tumors. A majority of the incisural tumors usually display some neurological symptoms owing to their localizations. However, not infrequently 
the tumors which originate at or adjacent to the tentorial edge are neurologically less manifest and afford many difficulties, above all localization of the tumor attachment. In such cases, roentgenological examination will give a clue to solving the difficulties in the diagnosis and makes it possible for the neurosurgeon to find an approach appropriate for each case.

Recently, we have had six cases of tumors of this region. Three were extracerebral and the others were intracerebellar, that is in the culmen and in the lobus quadrangularis. Surgically, the supratentorial approach is the first choice. The suboccipital approach does not seem to be adequate in these tumors. In some cases, however, the latter approach was also necessary following the former, that is, combined supra and infratentorial approach simultaneously. For the purpose of this approach, we employed the big hockey-stick shaped skin incision with the handle extending along the midline from the parietal to the nuchal region. The operative results were good. There was no operative death. Two cases had postoperative sequelae and the other four fully recovered.

\title{
e-19. Merits and Limitation of Microsurgery in the Neurosurgical Field
}

\author{
Hiroshi Matsumura, Yasumasa Makita, Yasuo Kihara, Ichizo Yoshimoto, \\ Hiroshi KaJIKawa, Terukuni IMaI and Juli TakeUCHI \\ Department of Neurosurgery, Tenri Hospital
}

Operative microscope which was developed in the otological field has also found more frequent application in the neurosurgical field, but not to the extent as previously expected. Merits and demerits of the operation under microscope will be discussed from our experiences. Our experiences of operation under microscope are showed in the table.

\section{Facilities:}

1) As the direction of the light and the gaze is the same, the microscopic technic is favorable for operation in deep place with narrow opening. Operative microscope has an advantage over head light equipment in this respect, though magnification of the image is not significant, because of no need of wide opening in the skull.

2) Bleeding from all capillaries can be satisfactorily controlled throughout the operative route when it is limited space; for example, dissection of interhemispheric space between both anterior lobes when anterior communicating aneurysm is attacked through interhemispheric route.

3) Distinction of the important nerves and vessels from tumor tissue is easy under microscope. For this reason microscope was indispensable in operation of translabyrinthine approach to acoustic neurinoma and intracranial section of the vestibular portion of the acoustic nerve for Mèniére's disease and some other 